\title{
A PLANE STRESS SOFTENING PLASTICITY MODEL FOR ORTHOTROPIC MATERIALS
}

\author{
PAULO B. LOURENÇO, ${ }^{1, * * * *}$ RENÉ DE BORST ${ }^{1,2}$ AND JAN G. ROTS ${ }^{1,3}$ \\ ${ }^{1}$ Delft University of Technology, Faculty of Civil Engineering, P.O. Box 5048, NL-2600 GA Delft, The Netherlands \\ ${ }^{2}$ Eindhoven University of Technology, Faculty of Mechanical Engineering, The Netherlands \\ ${ }^{3}$ TNO Building and Construction Research, P.O. Box 49, NL-2600 AA Delft, The Netherlands
}

\begin{abstract}
A plane stress model has been developed for quasi-brittle orthotropic materials. The theory of plasticity, which is adopted to describe the inelastic behaviour, utilizes modern algorithmic concepts, including an implicit Euler backward return mapping scheme, a local Newton-Raphson method and a consistent tangential stiffness matrix. The model is capable of predicting independent responses along the material axes. It features a tensile fracture energy and a compressive fracture energy, which are different for each material axis. A comparison between calculated and experimental results in masonry shear walls shows that a successful implementation has been achieved. (C) 1997 John Wiley \& Sons, Ltd.
\end{abstract}

Int. J. Numer. Meth. Engng., 40, 4033-4057 (1997)

No. of Figures: 13. No. of Tables: 4. No. of References: 28.

KEY WORDS: plasticity; orthotropy; fracture energy; plane stress; masonry

\section{INTRODUCTION}

A large number of anisotropic materials exist in engineering such as masonry, plastics, wood and most composites. The framework of plasticity theory is general enough to apply to both isotropic and anisotropic behaviour. Indeed, the past decade has witnessed numerous publications on sound numerical implementations of isotropic plasticity models. Nevertheless, it appears that, while some anisotropic plasticity models have been proposed from purely theoretical and experimental standpoints, ${ }^{1-3}$ only a few numerical implementations and calculations have actually been carried out. Examples include the work of de Borst and Feenstra ${ }^{4}$ and Schellekens and de Borst ${ }^{5}$ who fully treated the implementation of elastic-perfectly-plastic Hill and Hoffman criteria, respectively. In these publications hardening behaviour has been simulated with the fraction model of Besseling. ${ }^{6}$ More recently, linear tensorial hardening has been incorporated in the Hill criterion ${ }^{7}$ and linear hardening has been implemented in a modified (pressure dependent) von Mises criterion to fit either the uniaxial tensile or the compressive behaviour. ${ }^{8}$

\footnotetext{
* Correspondence to: P. B. Lourenço, School of Engineering, University of Minho, Azurém, P-4800 Guimarães, Portugal

** Current address: Department of Civil Engineering, School of Engineering, University of Minho, Azurém, P-4800 Guimarães, Portugal
}

Contract grant sponsor: Netherlands Technology Foundation (STW); Contract grant number: DCT 33.3052 


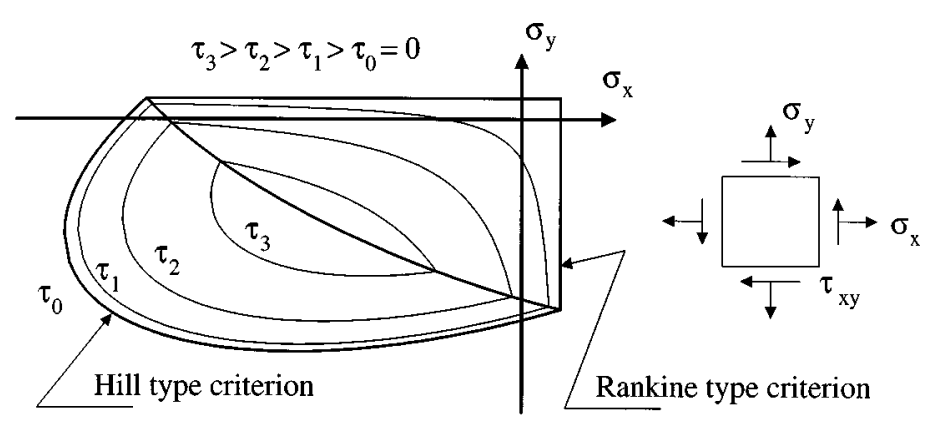

Figure 1. Proposed composite yield criterion with iso-shear stress lines. Different strength values for tension and compression are assumed along each material axis

It is not surprising that only a few anisotropic models have been implemented and tested successfully. An accurate analysis of anisotropic materials requires a description for all stress states. Difficulties arise due to the fact that few experimental results are generally available (for pre-peak and for post-peak behaviour), but also due to the intrinsic complexity of formulating anisotropic inelastic behaviour. The yield criterion proposed in the present study combines the advantages of modern plasticity concepts with a powerful representation of anisotropic material behaviour, which includes different hardening/softening behaviour along each material axis.

The composite yield criterion proposed here is suitable for the modelling of anisotropic materials under plane stress conditions and consists of an extension of conventional formulations for isotropic quasi-brittle materials to describe orthotropic behaviour. Individual yield criteria are considered for tension and compression, describing different failure mechanisms. The first criterion is associated with a localised fracture process, namely cracking of the material, and the second criterion is associated with a more distributed fracture process which is usually named crushing of the material. The yield criterion represents a step further in the work of Feenstra and de Borst, ${ }^{9}$ who utilised this approach for concrete with a Rankine and a Drucker-Prager criterion.

In order to model orthotropic material behaviour, we purpose a Hill-type* criterion for compression and a Rankine-type ${ }^{\dagger}$ criterion for tension, see Figure 1. The internal damage due to these failure mechanisms is represented with two internal parameters, one for damage in tension and one for damage in compression. The model is formulated in such a way that each internal parameter is related to two independent fracture energies along each material axis.

\section{DISCRETIZATION ASPECTS OF SOFTENING MATERIALS}

Early applications of finite element analysis to the mechanics of quasi-brittle materials adopted a sudden drop to a zero stress level upon violation of the tensile and compressive strength.

\footnotetext{
* The word type is used here because Hill ${ }^{1}$ adopted a three-dimensional formulation. In plane stress conditions, the influence of the out-of-plane direction is generally unknown and it will not be considered in this study. The yield criterion adopted should, in fact, be considered as a particular case of the complete quadratic formulation from $\mathrm{Tsai}^{\mathrm{and}} \mathrm{Wu}^{3}$ ${ }^{\dagger}$ The word type is used here because the Rankine criterion represents the material strength along the maximum principal stress. For an anisotropic material such definition is clearly not possible. The proposed yield criterion for tension will be derived from the original Rankine criterion but represents solely a fit of experimental results
} 
Carefully executed experiments using servo-controlled equipment soon revealed that this assumptions were erroneous, since a descending branch was observed. For this reason, 'softening' stress-strain relations were adopted, in which the stress was made a descending function of the strain. The assumption of a stress-strain relation directly related to the experiments resulted in analysis governed by the finite element mesh. This problem has been solved by relating the softening stress-strain relations to the element size. ${ }^{10-12}$

Note that no argument is given here about strain softening localisation and mesh dependency. The authors recognise that the problem of mesh objectivity is not completed solved by this 'energy' approach. But, for tensile cracking, the concept of energy dissipation, the fracture energy $G_{\mathrm{f}}$, has been extensively used in finite element calculations and can be considered as accepted in the engineering community. With the assumption that the fracture energy is uniformly dissipated in a representative area of the structure, the equivalent length $h$, the finite element calculations will lead to objective results with regard to mesh refinement. Thus, in the following, the softening behaviour will be modelled with a smeared approach in which the damaged material is still considered as a continuum. With this assumption, the localized damage can be represented by the scalar $\kappa$, which is related by an equivalent length to the released energy per unit cracked area, $G_{\mathrm{f}}$.

In the present article, the concept of released energy and equivalent length is also applied to model compressive behaviour by introducing a compressive fracture energy $G_{\mathrm{c}}$, although it is recognized that compressive failure is physically more a volume-driven process than a surfacedriven process. This equivalent concept for compressive loading ${ }^{13,14}$ is not a widespread idea in the engineering community but it is fundamental to obtain mesh objective results. ${ }^{9}$ It remains to be demonstrated if the compressive fracture energy is a material or a structural property, ${ }^{14}$ a consequence of the current limitations of computational tools to trace each and every tensile crack preceding the discontinuities at collapse, ${ }^{13}$ or a numerical artifice aimed at mesh insensitivity. $^{15}$

In a finite element calculation the equivalent length $h$, corresponds to a representative dimension of the mesh size so that the results obtained are objective with regard to mesh refinement. The equivalent length depends, at least, on the chosen element type, element size, element shape, integration scheme and even on the particular problem considered. In this study it is assumed that the equivalent length is related to the area of an element

$$
h=\alpha_{h} \sqrt{A_{e}}=\alpha_{h}\left(\sum_{\xi=1}^{n_{\xi}} \sum_{\eta=1}^{n_{\eta}} \operatorname{det}(\mathbf{J}) w_{\xi} w_{\eta}\right)^{1 / 2}
$$

in which $w_{\xi}$ and $w_{\eta}$ are the weight factors of the Gaussian integration rule as it is tacitly assumed that the elements are always integrated numerically. The local, isoparametric coordinates of the integration points are given by $\xi$ and $\eta$. The factor $\alpha_{h}$ is a modification factor which is equal to one for quadratic elements and equal to $\sqrt{2}$ for linear elements. For straining not parallel to the mesh lines, the above values give a good approximation. ${ }^{16}$

The inelastic work $g_{\mathrm{f} / \mathrm{c}}$ is defined by the integral

$$
g_{\mathrm{f} / \mathrm{c}}=\int \boldsymbol{\sigma}^{\mathrm{T}} \mathrm{d} \boldsymbol{\varepsilon}=\int \boldsymbol{\sigma}^{\mathrm{T}} \mathrm{d} \boldsymbol{\varepsilon}^{\mathrm{p}}
$$

which corresponds to the area under the stress-strain diagram for uniaxial loading. Assuming that the inelastic work $g_{\mathrm{f} / \mathrm{c}}$ is uniformly distributed over the equivalent length, the relation 
between the fracture energy $G_{\mathrm{f} / \mathrm{c}}$ and the work $g_{\mathrm{f} / \mathrm{c}}$ is given by

$$
g_{\mathrm{f} / \mathrm{c}}=\frac{G_{\mathrm{f} / \mathrm{c}}}{h}
$$

This results in a material model related to the energy which has to be dissipated due to the irreversible damage in the material.

\section{NUMERICAL IMPLEMENTATION OF THE PLASTICITY THEORY}

In this study the finite element method is adopted to compute the structural behaviour. A mathematical description of the material behaviour, which yields the relation between the stress and strain tensor in a material point of the body, is necessary for this purpose. Such a mathematical description is commonly named a constitutive model. The constitutive model developed here will be cast in a plasticity framework with a phenomenological approach in which the observed mechanisms are represented in such a fashion that simulations are in reasonable agreement with experiments. Indeed, it is presently not (yet) possible to formulate constitutive models which fully incorporate all the interacting mechanisms of a specific material. A simplified, macroscopic representation is then more desirable, since it is believed that more insight can be gained by tracing the entire response of a structure using a relatively simple model than modelling it with a highly sophisticated material model or theory which does not result in a converged solution close to and beyond the failure load.

The theory of plasticity is well established and sound numerical algorithms have been developed. ${ }^{17}$ It is a natural constitutive description for metals, but it can also be used for quasi-brittle cementitious materials loaded in triaxial compression and shear-compression problems where inelastic non-recoverable strains are observed. The incapability of the theory to reproduce the elastic stiffness degradation of quasi-brittle materials subjected mainly to tension cannot be accepted for cyclic loading. Nevertheless, good results have been found for monotonic loading conditions..$^{9,18}$

\subsection{Rate formulation}

A fundamental notion in the plasticity theory is the existence of a yield function that bounds the elastic domain. Yielding can only occur if the stresses $\sigma$ satisfy the general yield criterion

$$
f(\boldsymbol{\sigma}, \bar{\sigma}(\kappa))=0
$$

where the yield stress value $\bar{\sigma}$ is a function, commonly named hardening law, of the scalar $\kappa$, which is introduced as a measure for the amount of inelastic straining.

In general, it is very difficult to describe the material behaviour with a single yield surface in an appropriate manner and one usually has to resort to the theory of multisurface plasticity. In this case the elastic domain is defined by a number of functions $f_{i}<0$ which define a composite yield surface. Loading/unloading can be conveniently established in standard Kuhn-Tucker form by means of the conditions

$$
\dot{\lambda}_{i} \geqslant 0, \quad f_{i} \leqslant 0, \quad \dot{\lambda}_{i} f_{i}=0
$$


where $\dot{\lambda}_{i}$ is the plastic multiplier. Plastic behaviour is characterized by a non-unique stress-strain relation with the presence of irreversible strains on load removal. This is obtained by the usual decomposition of the strain rate vector $\dot{\boldsymbol{\varepsilon}}$ in an elastic, reversible part, $\dot{\boldsymbol{\varepsilon}}^{\mathrm{e}}$ and a plastic, irreversible part, $\dot{\boldsymbol{\varepsilon}}^{\mathrm{p}}$

$$
\dot{\boldsymbol{\varepsilon}}=\dot{\boldsymbol{\varepsilon}}^{\mathrm{e}}+\dot{\boldsymbol{\varepsilon}}^{\mathrm{p}}
$$

where the elastic strain rate is related to the stress rate by the elastic (constitutive) stiffness matrix D as

$$
\dot{\boldsymbol{\sigma}}=\mathbf{D} \dot{\boldsymbol{\varepsilon}}^{\mathrm{e}}
$$

and, for single surface plasticity, the assumption of a non-associated flow rule yields

$$
\dot{\boldsymbol{\varepsilon}}^{\mathrm{p}}=\dot{\lambda} \frac{\partial g}{\partial \boldsymbol{\sigma}}
$$

where $g$ is a plastic potential. For the definitions of inelastic behaviour adopted in the present article, it will be shown that a particularly simple expression is obtained, where

$$
\dot{\kappa}=\dot{\lambda}
$$

For multisurface plasticity corners exist at the intersection of the different yield surfaces. We adopt a composite yield surface that is defined by two yield surfaces. According to Koiter's generalization, ${ }^{19}$ the plastic strain rate vector $\dot{\boldsymbol{\varepsilon}}^{\mathrm{p}}$ in the corner is obtained from a linear combination of the plastic strain rates of the two yield surfaces,

$$
\dot{\boldsymbol{\varepsilon}}^{\mathrm{p}}=\dot{\boldsymbol{\varepsilon}}_{1}^{\mathrm{p}}+\dot{\boldsymbol{\varepsilon}}_{2}^{\mathrm{p}}=\dot{\lambda}_{1} \frac{\partial g_{1}}{\partial \boldsymbol{\sigma}}+\dot{\lambda}_{2} \frac{\partial g_{2}}{\partial \boldsymbol{\sigma}}
$$

The yield surfaces will be assumed uncoupled because they represent different failure mechanisms.

\subsection{Integration of the elastoplastic equations}

The integration of the rate equations is a problem of evolution that can be regarded as follows. At stage $n$ the total strain field and the plastic strain field as well as the hardening parameter(s) are known:

$$
\left\{\boldsymbol{\varepsilon}_{n}, \boldsymbol{\varepsilon}_{n}^{\mathrm{p}}, \kappa_{i, n}\right\}
$$

The elastic strain and stress fields are regarded as dependent variables which can always be obtained from the basic variables through the relations

$$
\boldsymbol{\varepsilon}_{n}^{\mathrm{e}}=\varepsilon_{n}-\varepsilon_{n}^{\mathrm{p}}, \quad \boldsymbol{\sigma}_{n}=\mathbf{D} \varepsilon_{n}^{\mathrm{e}}
$$

Therefore, the stress field at stage $n+1$ is computed once the strain field is known (in the following the subscript $n+1$ will be generally dropped for convenience in the derivatives). The problem is strain driven in the sense that the total strain vector $\varepsilon$ is trivially updated according to the exact formula

$$
\varepsilon_{n+1}=\varepsilon_{n}+\Delta \varepsilon_{n+1}
$$

It remains to update the plastic strains and the hardening parameter(s). These quantities are determined by integration of the flow rule(s) and hardening law(s) over the step $n \rightarrow n+1$. In the 
frame of an implicit Euler backward algorithm this problem is transformed into a constrained optimization problem governed by discrete Kuhn-Tucker conditions. ${ }^{17}$ The stability and accuracy of the Euler backward algorithm have been shown in different studies. ${ }^{4,5,20}$ For single surface plasticity, this algorithm results in the following set of nonlinear equations in the presence of yielding:

$$
\left\{\begin{array}{l}
\mathbf{D}^{-1}\left(\boldsymbol{\sigma}_{n+1}-\boldsymbol{\sigma}^{\text {trial }}\right)+\Delta \boldsymbol{\varepsilon}_{n+1}^{\mathrm{p}}=\mathbf{0} \\
f_{n+1}\left(\boldsymbol{\sigma}_{n+1}, \kappa_{n+1}\right)=0
\end{array}\right.
$$

in which $\Delta \boldsymbol{\varepsilon}_{n+1}^{\mathrm{p}}$ reads

$$
\Delta \boldsymbol{\varepsilon}_{n+1}^{\mathrm{p}}=\left.\Delta \lambda_{n+1} \frac{\partial g}{\partial \boldsymbol{\sigma}}\right|_{n+1}
$$

and the elastic predictor step returns the value of the elastic trial stress $\boldsymbol{\sigma}^{\text {trial }}$ as

$$
\boldsymbol{\sigma}^{\text {trial }}=\boldsymbol{\sigma}_{n}+\mathbf{D} \Delta \boldsymbol{\varepsilon}_{n+1}
$$

The unknowns of the system of nonlinear equations are the components of the stress vector $\boldsymbol{\sigma}_{n+1}$ plus the state variable $\Delta \kappa_{n+1}=\Delta \lambda_{n+1}$. The system is solved with a regular Newton-Raphson method.

For multisurface surface plasticity, the Euler backward algorithm results in the following set of nonlinear equations in the presence of yielding:

$$
\left\{\begin{array}{l}
\mathbf{D}^{-1}\left(\boldsymbol{\sigma}_{n+1}-\boldsymbol{\sigma}^{\text {trial }}\right)+\Delta \boldsymbol{\varepsilon}_{1, n+1}^{\mathrm{p}}+\Delta \boldsymbol{\varepsilon}_{2, n+1}^{\mathrm{p}}=\mathbf{0} \\
f_{1, n+1}\left(\boldsymbol{\sigma}_{n+1}, \kappa_{1, n+1}\right)=0 \\
f_{2, n+1}\left(\boldsymbol{\sigma}_{n+1}, \kappa_{2, n+1}\right)=0
\end{array}\right.
$$

in which $\Delta \boldsymbol{\varepsilon}_{1, n+1}^{\mathrm{p}}$ and $\Delta \boldsymbol{\varepsilon}_{2, n+1}^{\mathrm{p}}$ read

$$
\Delta \boldsymbol{\varepsilon}_{1, n+1}^{\mathrm{p}}=\left.\Delta \lambda_{1, n+1} \frac{\partial g_{1}}{\partial \boldsymbol{\sigma}}\right|_{n+1} \quad \Delta \boldsymbol{\varepsilon}_{2, n+1}^{\mathrm{p}}=\left.\Delta \lambda_{2, n+1} \frac{\partial g_{2}}{\partial \boldsymbol{\sigma}}\right|_{n+1}
$$

The unknowns of the system of nonlinear equations are the components of the stress vector $\boldsymbol{\sigma}_{n+1}$ plus the state variables $\Delta \kappa_{1, n+1}=\Delta \lambda_{1, n+1}$ and $\Delta \kappa_{2, n+1}=\Delta \lambda_{2, n+1}$. The system is again solved with a regular Newton-Raphson method.

The problem that remains is how to determine whether only one or both yield surfaces are active. The location of the corner, i.e. the intersection between the two yield surfaces, is unknown at the beginning of the load step and the trial stress cannot provide a sufficient criterion to determine which yield surfaces are active at the end of the load step. ${ }^{21}$ In the present study, a trial and error procedure is used to solve the return mapping. It is assumed that the set of initial active yield functions is the one defined by $f_{i}^{\text {trial }} \geqslant 0 .{ }^{17}$ If, after the return mapping is completed, any $\Delta \lambda_{i, n+1}<0$ or $f_{i, n+1}>0$ is found, the number of active yield surfaces is adjusted accordingly and the return mapping is restarted. During this process, one restart may be required or, rarely and only for larger increments, two restarts may be required before the correct number of active yield surfaces is obtained.

\subsection{Evaluation of the tangential stiffness matrix}

The linearization of the equations arising from the finite element discretization yields the tangential stiffness matrix, which plays a crucial role in the performance and the robustness of the 
Newton-Raphson method. It has been emphasized in the classical paper about consistent tangential operators for computational plasticity ${ }^{22}$ that the tangential stiffness matrix at structural level must be obtained by consistent linearization of the stress update resulting from the return mapping algorithm at the end of iteration $n+1$. According to equations (14) and (17) the return mapping algorithm contains as unknowns the components of the stress vector $\boldsymbol{\sigma}_{n+1}$ and some other state variables, namely $\Delta \kappa_{i, n+1}=\Delta \lambda_{i, n+1}$, which are conveniently collected in a vector of state variables $\mathbf{q}_{n+1}$. The number of components of $\mathbf{q}_{n+1}, n_{q}$, is one for single surface plasticity and two for the corner of a composite yield surface, cf. equations (14) and (17). Linearization of equations (14) or equations (17) then yields

$$
\mathbf{J}\left\{\begin{array}{c}
\mathrm{d} \boldsymbol{\sigma}_{n+1} \\
--- \\
\mathrm{d} \mathbf{q}_{n+1}
\end{array}\right\}=\left[\begin{array}{ccc}
\mathbf{J}_{n_{\sigma} \times n_{\sigma}} & \mid & \mathbf{J}_{n_{\sigma} \times n_{q}} \\
---- & + & ---- \\
\mathbf{J}_{n_{q} \times n_{\sigma}} & \mid & \mathbf{J}_{n_{q} \times n_{q}}
\end{array}\right]\left\{\begin{array}{c}
\mathrm{d} \boldsymbol{\sigma}_{n+1} \\
--- \\
\mathrm{d} \mathbf{q}_{n+1}
\end{array}\right)=\left\{\begin{array}{c}
\mathrm{d} \boldsymbol{\varepsilon}_{n+1} \\
--- \\
\mathbf{0}
\end{array}\right\}
$$

where $\mathbf{J}$ is the Jacobian calculated previously to solve the return mapping and $n_{\sigma}$ is the number of components of the stress vector $\boldsymbol{\sigma}$. Thus, the consistent tangential stiffness matrix $\mathbf{D}^{\mathrm{ep}}$ can simply be obtained as

$$
\mathbf{D}^{\mathrm{ep}}=\left.\frac{\mathrm{d} \boldsymbol{\sigma}_{n+1}}{\mathrm{~d} \boldsymbol{\varepsilon}_{n+1}}\right|_{n+1}=\left(\mathbf{J}^{-1}\right)_{n_{\sigma} \times n_{\sigma}}
$$

where $\left(\mathbf{J}^{-1}\right)_{n_{\sigma} \times n_{\sigma}}$ is the top-left $n_{\sigma} \times n_{\sigma}$ submatrix of the inverse of the Jacobian $\mathbf{J}$. This expression for the consistent tangential stiffness matrix is generally applicable and is economical for complex plasticity models since it employs the tangential stiffness matrix from the local Newton-Raphson method for the return mapping to construct the consistent tangential stiffness matrix for the Newton-Raphson procedure at structural level.

\section{THE PROPOSED MATERIAL MODEL}

\subsection{A Rankine-type criterion}

An adequate formulation of the Rankine criterion consists of a single function of the first principal stress and an equivalent stress $\bar{\sigma}_{\mathrm{t}}$ which describes the softening behaviour of the material $^{23}$

$$
f_{1}=\frac{\sigma_{x}+\sigma_{y}}{2}+\sqrt{\left(\frac{\sigma_{x}-\sigma_{y}}{2}\right)^{2}+\tau_{x y}^{2}}-\bar{\sigma}_{\mathrm{t}}\left(\kappa_{\mathrm{t}}\right)
$$

where the scalar $\kappa_{\mathrm{t}}$ controls the amount of softening. The assumption of isotropic softening is not completely valid for quasi-brittle materials which can be loaded up to the tensile strength even if damage has already occurred in the perpendicular direction. This problem can be solved partially by using a kinematic softening formulation where the yield surface is shifted in the direction of the first principal stress. ${ }^{23}$ However, if in a certain direction softening is completed and, subsequently the material is loaded in a direction orthogonal to the previous loading direction, ideally plastic behaviour is found in the new direction. This is because all the fracture energy has been consumed during the opening of the first crack. A possible solution is the use of two independent softening parameters to control the shifting of the yield surface. Such a formulation of the Rankine 
criterion, which reproduces exactly the material behaviour in tension, leads to a response that is between the fixed and rotating crack models. ${ }^{24}$ Therefore, it has the benefit of a model with memory and with a flexible shear response. Unfortunately, numerical difficulties ${ }^{18}$ preclude its use in large scale computations.

In this contribution, a single scalar is used to control an orthotropic Rankine type criterion. The scalar measures the amount of softening simultaneously in the two material axes, but the model still incorporates two different fracture energies. The expression for the Rankine criterion, cf. equation (21), is rewritten as

$$
f_{1}=\frac{\left(\sigma_{x}-\bar{\sigma}_{\mathrm{t}}\left(\kappa_{\mathrm{t}}\right)\right)+\left(\sigma_{y}-\bar{\sigma}_{\mathrm{t}}\left(\kappa_{\mathrm{t}}\right)\right)}{2}+\sqrt{\left(\frac{\left(\sigma_{x}-\bar{\sigma}_{\mathrm{t}}\left(\kappa_{\mathrm{t}}\right)\right)-\left(\sigma_{y}-\bar{\sigma}_{\mathrm{t}}\left(\kappa_{\mathrm{t}}\right)\right)}{2}\right)^{2}+\tau_{x y}^{2}}
$$

where coupling exists between the stress components and the yield value. Setting forth a Rankine-type yield surface for an orthotropic material, with different yield values along the $x, y$ directions $\bar{\sigma}_{\mathrm{t} x}$ and $\bar{\sigma}_{\mathrm{t} y}$, is now straightforward if equation (22) is modified to

$$
f_{1}=\frac{\left(\sigma_{x}-\bar{\sigma}_{\mathrm{t} x}\left(\kappa_{\mathrm{t}}\right)\right)+\left(\sigma_{y}-\bar{\sigma}_{\mathrm{t} y}\left(\kappa_{\mathrm{t}}\right)\right)}{2}+\sqrt{\left(\frac{\left(\sigma_{x}-\bar{\sigma}_{\mathrm{t} x}\left(\kappa_{\mathrm{t}}\right)\right)-\left(\sigma_{y}-\bar{\sigma}_{\mathrm{t} y}\left(\kappa_{\mathrm{t}}\right)\right)}{2}\right)^{2}+\alpha \tau_{x y}^{2}}
$$

where the parameter $\alpha$, which controls the shear stress contribution to failure, reads

$$
\alpha=\frac{f_{\mathrm{tx} x} f_{\mathrm{t} y}}{\tau_{u, \mathrm{t}}^{2}}
$$

Here, $f_{\mathrm{t} x}$ and $f_{\mathrm{t} y}$ and $\tau_{u, \mathrm{t}}$ are the uniaxial tensile strengths in the $x, y$ directions and the pure shear strength, respectively, see Figure 2. Note that the material axes are now fixed with respect to a specific frame of reference and it shall be assumed that all stresses and strains for the elastoplastic algorithm are given in the material reference axes, see also Section 4.4. Equation (23) can be, advantageously, recast in a matrix form as

$$
f_{1}=\left(\frac{1}{2} \xi^{\mathrm{T}} \mathbf{P}_{\mathrm{t}} \xi\right)^{1 / 2}+\frac{1}{2} \pi^{\mathrm{T}} \xi
$$

where the projection matrix $\mathbf{P}_{\mathrm{t}}$ reads

$$
\mathbf{P}_{\mathrm{t}}=\left[\begin{array}{rrc}
\frac{1}{2} & -\frac{1}{2} & 0 \\
-\frac{1}{2} & \frac{1}{2} & 0 \\
0 & 0 & 2 \alpha
\end{array}\right]
$$

the projection vector $\pi$ reads

$$
\pi=\left\{\begin{array}{lll}
1 & 1 & 0
\end{array}\right\}^{\mathrm{T}}
$$

and the reduced stress vector $\xi$ reads

$$
\xi=\sigma-\eta
$$

The stress vector $\boldsymbol{\sigma}$ and the back stress vector $\boldsymbol{\eta}$ are given by

$$
\begin{aligned}
& \boldsymbol{\sigma}=\left\{\begin{array}{lll}
\sigma_{x} & \sigma_{y} & \tau_{x y}
\end{array}\right\}^{\mathrm{T}} \\
& \boldsymbol{\eta}=\left\{\begin{array}{lll}
\bar{\sigma}_{\mathrm{t} x}\left(\kappa_{\mathrm{t}}\right) & \bar{\sigma}_{\mathrm{ty}}\left(\kappa_{\mathrm{t}}\right) & 0
\end{array}\right\}^{\mathrm{T}}
\end{aligned}
$$




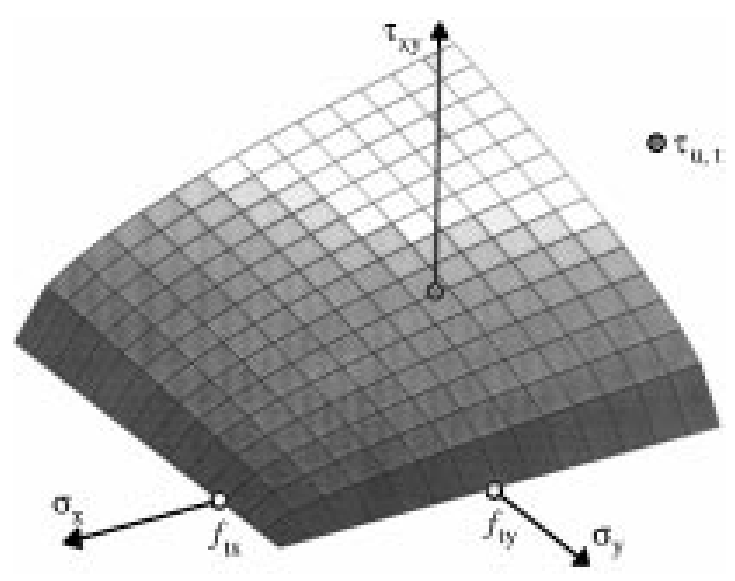

Figure 2. Orthotropic Rankine-type criterion (shown for $\tau_{x y} \geqslant 0$ )

Exponential tensile softening is considered for both equivalent stress-equivalent strain diagrams, with different fracture energies $\left(G_{\mathrm{f} x}\right.$ and $\left.G_{\mathrm{f} y}\right)$ for each yield value, such that

$$
\bar{\sigma}_{\mathrm{t} x}=f_{\mathrm{t} x} \exp \left(-\frac{h f_{\mathrm{t} x}}{G_{\mathrm{f} x}} \kappa_{\mathrm{t}}\right), \quad \bar{\sigma}_{\mathrm{t} y}=f_{\mathrm{t} y} \exp \left(-\frac{h f_{\mathrm{t} y}}{G_{\mathrm{f} y}} \kappa_{\mathrm{t}}\right)
$$

It is noted that the equivalent length $h$, cf. equation (1), can lead to snap-back behaviour at constitutive level if the element size is large. Then, the concept of fracture energy which has been assumed is no longer satisfied. In such a case, the strength limit would have to be reduced in order to obtain an objective fracture energy. To prevent this from happening the equivalent length $h$ is bounded by

$$
h \leqslant \frac{G_{\mathrm{fi}} E_{i}}{f_{\mathrm{t} i}^{2}}
$$

where $E_{i}$ is the Young's modulus and the subscript $i$ refers to the material axis.

A non-associated plastic potential $g_{1}$

$$
g_{1}=\left(\frac{1}{2} \xi^{\mathrm{T}} \mathbf{P}_{\mathrm{g}} \xi\right)^{1 / 2}+\frac{1}{2} \pi^{\mathrm{T}} \xi
$$

is considered, where the projection matrix $\mathbf{P}_{\mathrm{g}}$ represents the original Rankine plastic flow and reads

$$
\mathbf{P}_{\mathrm{g}}=\left[\begin{array}{rrr}
\frac{1}{2} & -\frac{1}{2} & 0 \\
-\frac{1}{2} & \frac{1}{2} & 0 \\
0 & 0 & 2
\end{array}\right]
$$

Note that for $\alpha=1, \mathbf{P}_{\mathbf{t}}=\mathbf{P}_{\mathrm{g}}$. The description of the inelastic behaviour is then completed by a softening hypothesis which involves the maximum principal plastic strain $\dot{\varepsilon}_{1}^{\text {p }}$

$$
\dot{\kappa}_{\mathrm{t}}=\dot{\varepsilon}_{1}^{\mathrm{p}}=\frac{\dot{\varepsilon}_{x}^{\mathrm{p}}+\dot{\varepsilon}_{y}^{\mathrm{p}}}{2}+\frac{1}{2} \sqrt{\left(\dot{\varepsilon}_{x}^{\mathrm{p}}-\dot{\varepsilon}_{y}^{\mathrm{p}}\right)^{2}+\left(\dot{\gamma}_{x y}^{\mathrm{p}}\right)^{2}}
$$


This expression can be recast in a matrix form as

$$
\dot{\kappa}_{\mathrm{t}}=\dot{\varepsilon}_{1}^{\mathrm{p}}=\left(\frac{1}{2}\left(\dot{\boldsymbol{\varepsilon}}^{\mathrm{p}}\right)^{\mathrm{T}} \mathbf{Q} \dot{\boldsymbol{\varepsilon}}^{\mathrm{p}}\right)^{1 / 2}+\frac{1}{2} \pi^{\mathrm{T}} \dot{\boldsymbol{\varepsilon}}^{\mathrm{p}}
$$

where

$$
\mathbf{Q}=\left[\begin{array}{rrr}
\frac{1}{2} & -\frac{1}{2} & 0 \\
-\frac{1}{2} & \frac{1}{2} & 0 \\
0 & 0 & \frac{1}{2}
\end{array}\right]
$$

which reduces to the particularly simple expression, cf. equation (9),

$$
\dot{\kappa}_{\mathrm{t}}=\dot{\lambda}_{\mathrm{t}}
$$

The return mapping algorithm, cf. equation (14), reduces to the following set of four equations which contain four unknowns (the components of $\sigma_{n+1}$ and $\Delta \kappa_{\mathrm{t}, n+1}=\Delta \lambda_{\mathrm{t}, n+1}$ )

$$
\left\{\begin{array}{l}
\mathbf{D}^{-1}\left(\boldsymbol{\sigma}_{n+1}-\boldsymbol{\sigma}^{\text {trial }}\right)+\left.\Delta \lambda_{\mathrm{t}, n+1} \frac{\partial g_{1}}{\partial \boldsymbol{\sigma}}\right|_{n+1}=\mathbf{0} \\
f_{1, n+1}=\left(\frac{1}{2} \xi_{n+1}^{\mathrm{T}} \mathbf{P}_{\mathrm{t}} \xi_{n+1}\right)^{1 / 2}+\frac{1}{2} \pi^{\mathrm{T}} \xi_{n+1}=0
\end{array}\right.
$$

where $\mathbf{D}$ represents the symmetric orthotropic elasticity tensor. The Jacobian necessary for the iterative local Newton-Raphson method can be elaborated as

$$
\mathbf{J}_{n+1}=\left[\begin{array}{ccc}
\mathbf{D}^{-1}+\Delta \lambda_{\mathrm{t}, n+1} \frac{\partial^{2} g_{1}}{\partial \boldsymbol{\sigma}^{2}} & \mid & \frac{\partial g_{1}}{\partial \boldsymbol{\sigma}}+\Delta \lambda_{\mathrm{t}, n+1} \frac{\partial^{2} g_{1}}{\partial \boldsymbol{\sigma} \partial \kappa_{\mathrm{t}}} \\
--------- & + & ---------- \\
\left(\frac{\partial f_{1}}{\partial \boldsymbol{\sigma}}\right)^{\mathrm{T}} & \mid & \frac{\partial f_{1}}{\partial \kappa_{\mathrm{t}}}
\end{array}\right]
$$

where

$$
\begin{aligned}
\frac{\partial f_{1}}{\partial \boldsymbol{\sigma}} & =\frac{\mathbf{P}_{\mathrm{t}} \xi_{n+1}}{2\left(\frac{1}{2} \xi_{n+1}^{\mathrm{T}} \mathbf{P}_{\mathrm{t}} \xi_{n+1}\right)^{1 / 2}}+\frac{1}{2} \boldsymbol{\pi} \\
\frac{\partial g_{1}}{\partial \boldsymbol{\sigma}} & =\frac{\mathbf{P}_{\mathrm{g}} \xi_{n+1}}{2\left(\frac{1}{2} \xi_{n+1}^{\mathrm{T}} \mathbf{P}_{\mathrm{g}} \xi_{n+1}\right)^{1 / 2}}+\frac{1}{2} \boldsymbol{\pi} \\
\frac{\partial f_{1}}{\partial \kappa_{\mathrm{t}}} & =-\left(\frac{\partial f_{1}}{\partial \boldsymbol{\sigma}}\right)^{\mathrm{T}} \frac{\partial \boldsymbol{\eta}}{\partial \kappa_{\mathrm{t}}} \\
\frac{\partial \boldsymbol{\eta}}{\partial \kappa_{\mathrm{t}}} & =\left\{\frac{\partial \bar{\sigma}_{\mathrm{t} x}}{\partial \kappa_{\mathrm{t}}}, \frac{\partial \bar{\sigma}_{\mathrm{ty}}}{\partial \kappa_{\mathrm{t}}}, 0\right\}^{\mathrm{T}} \\
\frac{\partial^{2} g_{1}}{\partial \boldsymbol{\sigma}^{2}} & =\frac{\mathbf{P}_{\mathrm{g}}}{2\left(\frac{1}{2} \xi_{n+1}^{\mathrm{T}} \mathbf{P}_{\mathrm{g}} \xi_{n+1}\right)^{1 / 2}}-\frac{\mathbf{P}_{\mathrm{g}} \xi_{n+1} \xi_{n+1}^{\mathrm{T}} \mathbf{P}_{\mathrm{g}}}{4\left(\frac{1}{2} \xi_{n+1}^{\mathrm{T}} \mathbf{P}_{\mathrm{g}} \xi_{n+1}\right)^{3 / 2}} \\
\frac{\partial^{2} g_{1}}{\partial \boldsymbol{\sigma} \partial \kappa_{\mathrm{t}}} & =-\frac{\partial^{2} g_{1}}{\partial \boldsymbol{\sigma}^{2}} \frac{\partial \boldsymbol{\eta}}{\partial \kappa_{\mathrm{t}}}
\end{aligned}
$$


The above algorithm is not stable for the entire stress domain because the gradient of the plastic potential in the apex, cf. equation $(40)_{2}$, is not defined. Nevertheless, a quite simple algorithm can be used for the apex regime because $\tau_{x y}=0$. In this case, the stress update, cf. equation $(38)_{1}$, is simply a return mapping to the apex, independently of the trial stress value, and reads

$$
\boldsymbol{\sigma}_{n+1}=\boldsymbol{\eta}_{n+1}
$$

The above stress update is sufficient to fulfill $f_{1, n+1}=0$. It remains to update the softening scalar according to equation (35). For this purpose a non-linear equation can be setup as follows:

$$
F\left(\Delta \kappa_{\mathrm{t}, n+1}\right)=\Delta \kappa_{\mathrm{t}, n+1}-\Delta \varepsilon_{1, n+1}^{\mathrm{p}}=\Delta \kappa_{\mathrm{t}, n+1}-\left(\frac{1}{2}\left(\Delta \boldsymbol{\varepsilon}_{n+1}^{\mathrm{p}}\right)^{\mathrm{T}} \mathbf{Q} \Delta \boldsymbol{\varepsilon}_{n+1}^{\mathrm{p}}\right)^{1 / 2}+\frac{1}{2} \pi^{\mathrm{T}} \Delta \boldsymbol{\varepsilon}_{n+1}^{\mathrm{p}}=0
$$

where the increment of the plastic strain vector $\Delta \boldsymbol{\varepsilon}_{n+1}^{\mathrm{p}}$ can be calculated from

$$
\Delta \varepsilon_{n+1}^{\mathrm{p}}=\mathbf{D}^{-1}\left(\boldsymbol{\sigma}^{\text {trial }}-\boldsymbol{\sigma}_{n+1}\right)
$$

The consistent tangential stiffness matrix is directly obtained from equation (20) for the standard regime. For the apex, differentiation of the update equations yields, after algebraic manipulation,

$$
\mathbf{D}^{\mathrm{ep}}=\left.\frac{\partial \boldsymbol{\sigma}}{\partial \boldsymbol{\varepsilon}}\right|_{n+1}=\left[\mathbf{I}+\frac{\partial \boldsymbol{\eta}}{\partial \kappa_{\mathrm{t}}}\left(\frac{\partial \Delta \kappa_{\mathrm{t}}}{\partial \boldsymbol{\varepsilon}^{\mathrm{p}}}\right)^{\mathrm{T}} \mathbf{D}^{-1}\right]^{-1} \frac{\partial \boldsymbol{\eta}}{\partial \kappa_{\mathrm{t}}}\left(\frac{\partial \Delta \kappa_{\mathrm{t}}}{\partial \boldsymbol{\varepsilon}^{\mathrm{p}}}\right)^{\mathrm{T}}
$$

with

$$
\frac{\partial \Delta \kappa_{\mathrm{t}}}{\partial \boldsymbol{\varepsilon}^{\mathrm{p}}}=\frac{\mathbf{Q} \Delta \boldsymbol{\varepsilon}_{n+1}^{\mathrm{p}}}{2\left(\frac{1}{2}\left(\Delta \boldsymbol{\varepsilon}_{n+1}^{\mathrm{p}}\right)^{\mathrm{T}} \mathbf{Q} \Delta \boldsymbol{\varepsilon}_{n+1}^{\mathrm{p}}\right)^{1 / 2}}+\frac{1}{2} \pi
$$

\subsection{A Hill-type criterion}

The simplest yield surface that features different compressive strengths $\left(f_{\mathrm{c} x}\right.$ and $\left.f_{\mathrm{c} y}\right)$ along the material axes is a rotated centered ellipsoid in the full plane stress space $\left(\sigma_{x}, \sigma_{y}\right.$ and $\left.\tau_{x y}\right)$, see Figure 3. The expression for such a quadric is

$$
f_{2}=A \sigma_{x}^{2}+B \sigma_{x} \sigma_{y}+C \sigma_{y}^{2}+D \tau_{x y}^{2}-1=0
$$

where $A, B, C$ and $D$ are four material parameters such that $B^{2}-4 A C<0$, in order to ensure convexity.

For the numerical implementation the yield function can be advantageously recast in a square root matrix form and the variables can be defined in a more amenable way. For this purpose we first introduce the additional material parameters $\beta$ and $\gamma$ which determine the shape of the yield surface. The parameter $\beta$ controls the coupling between the normal stress values, i.e. rotates the yield surface around the shear stress axis, and must be obtained from one additional experimental test, e.g. a biaxial compression test with a unit ratio between the principal stresses. If this test is used to obtain the parameter $\beta$, the collapse load under biaxial compression $\left(\sigma_{x}=\sigma_{y}=-f_{45^{\circ}}\right.$ and $\tau_{x y}=0$ ) leads to

$$
\beta=\left[\frac{1}{f_{45^{\circ}}^{2}}-\frac{1}{f_{\mathrm{c} x}^{2}}-\frac{1}{f_{\mathrm{c} y}^{2}}\right] f_{\mathrm{c} x} f_{\mathrm{c} y}
$$




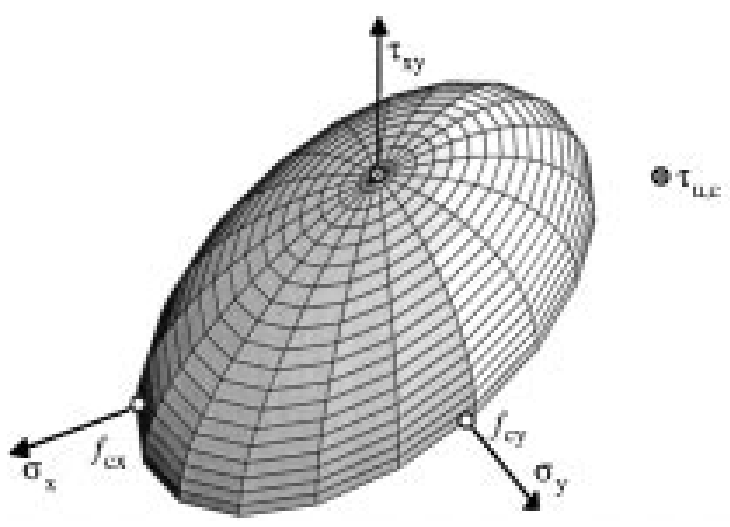

Figure 3. The Hill-type criterion (shown for $\tau_{x y} \geqslant 0$ )

The parameter $\gamma$, which controls the shear stress contribution to failure, can be obtained from

$$
\gamma=\frac{f_{\mathrm{cx}} f_{\mathrm{c} y}}{\tau_{u, \mathrm{c}}^{2}}
$$

where $\tau_{u, \mathrm{c}}$ is the material pure shear strength according to this criterion. With the definition of

$$
\begin{array}{ll}
A=1 /\left(\bar{\sigma}_{\mathrm{c} x}\left(\kappa_{\mathrm{c}}\right)\right)^{2}, & B=\beta /\left(\bar{\sigma}_{\mathrm{c} x}\left(\kappa_{\mathrm{c}}\right) \bar{\sigma}_{\mathrm{c} y}\left(\kappa_{\mathrm{c}}\right)\right) \\
C=1 /\left(\bar{\sigma}_{\mathrm{c} y}\left(\kappa_{\mathrm{c}}\right)\right)^{2}, & D=\gamma /\left(\bar{\sigma}_{\mathrm{c} x}\left(\kappa_{\mathrm{c}}\right) \bar{\sigma}_{\mathrm{c} y}\left(\kappa_{\mathrm{c}}\right)\right)
\end{array}
$$

where $\bar{\sigma}_{\mathrm{c} x}\left(\kappa_{\mathrm{c}}\right)$ and $\bar{\sigma}_{\mathrm{c} y}\left(\kappa_{\mathrm{c}}\right)$ are, respectively, the yield values along the material axes $x$ and $y$, the proposed yield surface can then be rewritten as

$$
f_{2}=\left(\frac{1}{2} \boldsymbol{\sigma}^{\mathrm{T}} \mathbf{P}_{\mathrm{c}} \boldsymbol{\sigma}\right)^{1 / 2}-\bar{\sigma}_{\mathrm{c}}\left(\kappa_{\mathrm{c}}\right)
$$

where the projection matrix $\mathbf{P}_{\mathrm{c}}$ reads

$$
\mathbf{P}_{\mathrm{c}}=\left[\begin{array}{ccc}
2 \frac{\bar{\sigma}_{\mathrm{c} y}\left(\kappa_{\mathrm{c}}\right)}{\bar{\sigma}_{\mathrm{c} x}\left(\kappa_{\mathrm{c}}\right)} & \beta & 0 \\
\beta & 2 \frac{\bar{\sigma}_{\mathrm{c} x}\left(\kappa_{\mathrm{c}}\right)}{\bar{\sigma}_{\mathrm{c} y}\left(\kappa_{\mathrm{c}}\right)} & 0 \\
0 & 0 & 2 \gamma
\end{array}\right]
$$

and $\bar{\sigma}_{\mathrm{c}}$ is given by

$$
\bar{\sigma}_{\mathrm{c}}=\sqrt{\bar{\sigma}_{\mathrm{c} x} \bar{\sigma}_{\mathrm{c} y}}
$$

while the scalar $\kappa_{\mathrm{c}}$ controls the amount of hardening and softening. The adopted inelastic law features parabolic hardening followed by parabolic/exponential softening and is used for both equivalent stress-equivalent strain diagrams, with different compressive fracture energies $\left(G_{\mathrm{fc} x}\right.$ and $G_{\mathrm{fcy}}$ ) along the material axes. The problem of mesh objectivity for analyses with strain softening materials is an intensely debated issue, at least for tensile behaviour, and in the present approach the stress-strain diagram is adjusted according to the characteristic length $h$ introduced 
in equation (1) to provide an objective energy dissipation. The inelastic law shown in Figure 4 features hardening, softening and a residual plateau of ideally plastic behaviour. The value of the compressive fracture energy $G_{\mathrm{fc} i}$ (shaded area in Figure 4) corresponds only to the local contribution of the $\bar{\sigma}_{\mathrm{c} i}-\kappa_{\mathrm{c}}$ diagram, where the subscript $i$ refers to the material axis. The basis for the above definition is numerical, in order to obtain objective results with regard to mesh refinement. ${ }^{18}$

The value for the peak strength is assumed to be reached simultaneously for both materials axes, i.e. isotropic hardening, followed by anisotropic softening governed by the different fracture energies. A residual strength value is adopted to avoid a cumbersome code (precluding the case when the compressive mode falls completely inside the tension mode) and to achieve a more robust code (precluding degeneration of the yield surface to a point). For practical reasons, it is assumed that all the stress values for the inelastic law are determined from the peak value $\bar{\sigma}_{\mathrm{p} i}=f_{\mathrm{c} i}$ as follows: $\bar{\sigma}_{i i}=\frac{1}{3} f_{\mathrm{c} i}, \bar{\sigma}_{\mathrm{m} i}=\frac{1}{2} f_{\mathrm{c} i}$ and $\bar{\sigma}_{\mathrm{r} i}=\frac{1}{10} f_{\mathrm{c} i}$. The equivalent plastic strain $\kappa_{\mathrm{p}}$ corresponding to the peak compressive strength is assumed to be an additional material parameter. In order to obtain a mesh independent energy dissipation the parameter $\kappa_{\mathrm{m} i}$ (see Figure 4) is given by

$$
\kappa_{\mathrm{m} i}=\frac{75}{67} \frac{G_{\mathrm{fc} i}}{h f_{\mathrm{c} i}}+\kappa_{\mathrm{p}}
$$

To avoid a possible snap-back at constitutive level, the condition

$$
\kappa_{\mathrm{m} i} \geqslant \frac{f_{\mathrm{c} i}}{E_{i}}+\kappa_{\mathrm{p}}
$$

is required.

An associated flow rule and a work-like hardening/softening hypothesis finally yield, cf. equation (9),

$$
\dot{\kappa}_{\mathrm{c}}=\frac{1}{\bar{\sigma}_{\mathrm{c}}} \boldsymbol{\sigma}^{\mathrm{T}} \dot{\boldsymbol{\varepsilon}}^{\mathrm{p}}=\dot{\lambda}_{\mathrm{c}}
$$

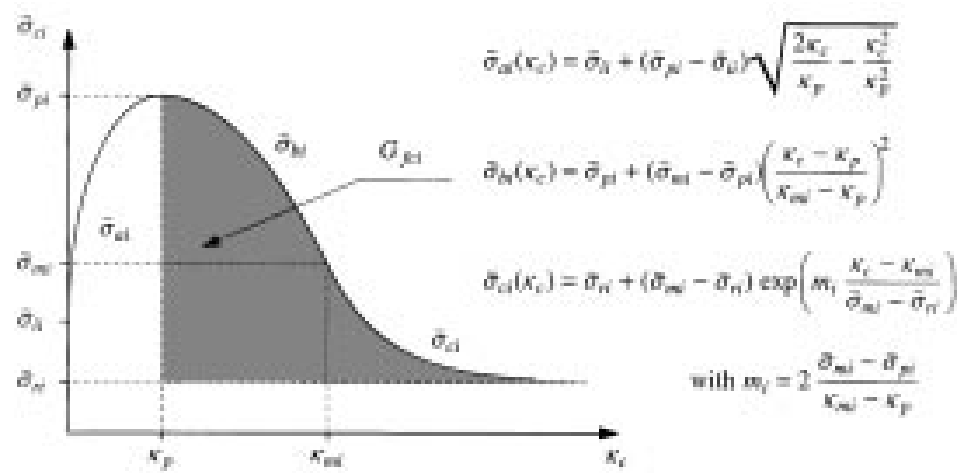

Figure 4. Hardening/softening law for compression 
The return mapping algorithm, cf. equation (14), reduces to the following set of four equations which contain four unknowns (the components of $\boldsymbol{\sigma}_{n+1}$ and $\Delta \kappa_{\mathrm{c}, n+1}=\Delta \lambda_{\mathrm{c}, n+1}$ )

$$
\left\{\begin{array}{l}
\mathbf{D}^{-1}\left(\boldsymbol{\sigma}_{n+1}-\boldsymbol{\sigma}^{\text {trial }}\right)+\left.\Delta \lambda_{\mathrm{c}, n+1} \frac{\partial f_{2}}{\partial \boldsymbol{\sigma}}\right|_{n+1}=\mathbf{0} \\
f_{2, n+1}=\left(\frac{1}{2} \boldsymbol{\sigma}_{n+1}^{\mathrm{T}} \mathbf{P}_{\mathrm{c}, n+1} \boldsymbol{\sigma}_{n+1}\right)^{1 / 2}-\bar{\sigma}_{\mathrm{c}, n+1}=0
\end{array}\right.
$$

The set of equations can be reduced to one nonlinear equation, ${ }^{25}$ namely $f_{2}\left(\Delta \lambda_{\mathrm{c}, n+1}\right)=0$. This approach will not be followed here in order to obtain a consistent framework for the several modes of the composite yield surface. Alternatively, the system of four non-linear equations is solved with a regular Newton-Raphson method. The Jacobian necessary for this procedure reads

$$
\mathbf{J}_{n+1}=\left[\begin{array}{ccc}
\mathbf{D}^{-1}+\Delta \lambda_{\mathrm{c}, n+1} \frac{\partial^{2} f_{2}}{\partial \boldsymbol{\sigma}^{2}} & \mid & \frac{\partial f_{2}}{\partial \boldsymbol{\sigma}}+\Delta \lambda_{\mathrm{c}, n+1} \frac{\partial^{2} f_{2}}{\partial \boldsymbol{\sigma} \partial \kappa_{\mathrm{c}}} \\
--------- & + & -------- \\
\left(\frac{\partial f_{2}}{\partial \boldsymbol{\sigma}}\right)^{\mathrm{T}} & \mid & \frac{\partial f_{2}}{\partial \kappa_{\mathrm{c}}}
\end{array}\right]
$$

where

$$
\left.\begin{array}{rl}
\frac{\partial f_{2}}{\partial \boldsymbol{\sigma}} & =\frac{\mathbf{P}_{\mathrm{c}} \boldsymbol{\sigma}_{n+1}}{2\left(\frac{1}{2} \boldsymbol{\sigma}_{n+1}^{\mathrm{T}} \mathbf{P}_{\mathrm{c}} \boldsymbol{\sigma}_{n+1}\right)^{1 / 2}} \\
\frac{\partial^{2} f_{2}}{\partial \boldsymbol{\sigma}^{2}} & =\frac{\mathbf{P}_{\mathrm{c}}}{2\left(\frac{1}{2} \boldsymbol{\sigma}_{n+1}^{\mathrm{T}} \mathbf{P}_{\mathrm{c}} \boldsymbol{\sigma}_{n+1}\right)^{1 / 2}}-\frac{\mathbf{P}_{\mathrm{c}} \boldsymbol{\sigma}_{n+1} \boldsymbol{\sigma}_{n+1}^{\mathrm{T}} \mathbf{P}_{\mathrm{c}}}{4\left(\frac{1}{2} \boldsymbol{\sigma}_{n+1}^{\mathrm{T}} \mathbf{P}_{\mathrm{c}} \boldsymbol{\sigma}_{n+1}\right)^{3 / 2}} \\
\frac{\partial f_{2}}{\partial \kappa_{\mathrm{c}}} & =\frac{\boldsymbol{\sigma}_{n+1}^{\mathrm{T}}\left(\partial \mathbf{P}_{\mathrm{c}} / \partial \kappa_{\mathrm{c}}\right) \boldsymbol{\sigma}_{n+1}}{4\left(\frac{1}{2} \boldsymbol{\sigma}_{n+1}^{\mathrm{T}} \mathbf{P}_{\mathrm{c}} \boldsymbol{\sigma}_{n+1}\right)^{1 / 2}}-\frac{\frac{\partial \bar{\sigma}_{\mathrm{c} x}}{\partial \kappa_{\mathrm{c}}} \bar{\sigma}_{\mathrm{c} y, n+1}+\frac{\partial \bar{\sigma}_{\mathrm{c} y}}{\partial \kappa_{\mathrm{c}}} \bar{\sigma}_{\mathrm{c} x, n+1}}{2 \bar{\sigma}_{\mathrm{c}, n+1}} \\
\frac{\partial^{2} f_{2}}{\partial \boldsymbol{\sigma} \partial \kappa_{\mathrm{c}}} & \left.\left.=\frac{\left(\partial \mathbf{P}_{\mathrm{c}} / \partial \kappa_{\mathrm{c}}\right) \boldsymbol{\sigma}_{n+1}}{2\left(\frac{1}{2} \boldsymbol{\sigma}_{n+1}^{\mathrm{T}} \mathbf{P}_{\mathrm{c}} \boldsymbol{\sigma}_{n+1}\right)^{1 / 2}}-\frac{\left(\boldsymbol{\sigma}_{n+1}^{\mathrm{T}}\left(\partial \mathbf{P}_{\mathrm{c}} / \partial \kappa_{\mathrm{c}}\right) \boldsymbol{\sigma}_{n+1}\right) \mathbf{P}_{\mathrm{c}} \boldsymbol{\sigma}_{n+1}}{8\left(\frac{1}{2} \boldsymbol{\sigma}_{n+1}^{\mathrm{T}} \mathbf{P}_{\mathrm{c}} \boldsymbol{\sigma}_{n+1}\right)^{1 / 2}}\right], 2\left[\frac{\partial \bar{\sigma}_{\mathrm{c} y}}{\partial \kappa_{\mathrm{c}}} \bar{\sigma}_{\mathrm{c} x, n+1}\right], 0\right\} \\
\frac{\partial \mathbf{P}_{\mathrm{c}}}{\partial \kappa_{\mathrm{c}}} & =\operatorname{diag}\left\{2\left[\frac{\partial \bar{\sigma}_{\mathrm{c} x, n+1}}{\partial \kappa_{\mathrm{c}}} \bar{\sigma}_{\mathrm{c} y, n+1}^{2}\right]\right.
\end{array}\right]
$$

The consistent tangential stiffness matrix is directly obtained from equation (20).

\subsection{A composite yield criterion}

The tension and compression yield criteria are now combined in a composite yield surface. Assuming that the tension and compression regimes are uncoupled, the return mapping 
algorithm for the corner regime, cf. equation (17), reduces to the following set of five equations which contain five unknowns (the components of $\sigma_{n+1}, \Delta \kappa_{\mathrm{t}, n+1}=\Delta \lambda_{\mathrm{t}, n+1}$ and $\left.\Delta \kappa_{\mathrm{c}, n+1}=\Delta \lambda_{\mathrm{c}, n+1}\right)$

$$
\left\{\begin{array}{l}
\mathbf{D}^{-1}\left(\boldsymbol{\sigma}_{n+1}-\boldsymbol{\sigma}^{\text {trial }}\right)+\left.\Delta \lambda_{\mathrm{t}, n+1} \frac{\partial g_{1}}{\partial \boldsymbol{\sigma}}\right|_{n+1}+\left.\Delta \lambda_{\mathrm{c}, n+1} \frac{\partial f_{2}}{\partial \boldsymbol{\sigma}}\right|_{n+1}=\mathbf{0} \\
f_{1, n+1}=\left(\frac{1}{2} \xi_{n+1}^{\mathrm{T}} \mathbf{P}_{\mathrm{t}} \xi_{n+1}\right)^{1 / 2}+\frac{1}{2} \pi^{\mathrm{T}} \xi_{n+1}=0 \\
f_{2, n+1}=\left(\frac{1}{2} \boldsymbol{\sigma}_{n+1}^{\mathrm{T}} \mathbf{P}_{\mathrm{c}, n+1} \boldsymbol{\sigma}_{n+1}\right)^{1 / 2}-\bar{\sigma}_{\mathrm{c}, n+1}=0
\end{array}\right.
$$

The Jacobian necessary for the iterative local Newton-Raphson method reads

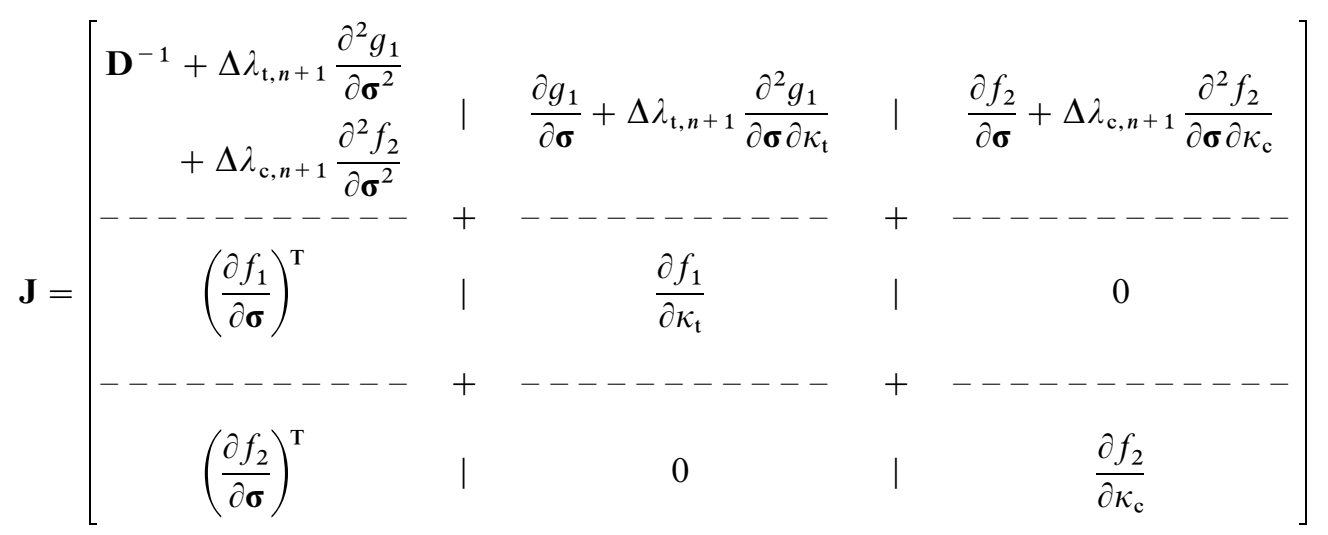

where all the terms have been defined in equations (40) and (58). The consistent tangential stiffness matrix is again directly obtained from equation (20).

\subsection{The orientation of the material axes}

For the sake of simplicity, the formulation of the plasticity model was presented based on the assumption that the principal axes of orthotropy coincided with the frame of reference (local or global) for stresses and strains in finite element computations. Since this is not necessarily the case, such non-alignment effects must be taken into account.

Two different approaches can be followed. In the first approach, with each call to the plasticity model, stresses, strains and, finally, consistent tangential stiffness matrices must be rotated into and out of the material frame of reference, respectively, as pre- and post-processing. In the second approach, before the analysis begins, the elastic stiffness matrix $\mathbf{D}$, the projection matrices $\mathbf{P}_{\mathrm{t}}, \mathbf{P}_{\mathrm{g}}$ and $\mathbf{P}_{\mathrm{c}}$, and the projection vectors $\boldsymbol{\pi}$ and $\boldsymbol{\eta}$ must be rotated from the material frame of reference into the global frame of reference at each quadrature point, eliminating the need of all subsequent rotation operations. The drawback of the latter approach is that the matrices then lose their sparse nature, which tends to make algorithm developments less clear and less efficient. For this reason, the plasticity model is implemented employing the former option. 


\section{THE INELASTIC BEHAVIOUR OF THE MODEL: ELEMENTARY TESTS}

This section provides insight into the inelastic behaviour of the anisotropic composite yield criterion. This is a relevant issue given the limited experience in using anisotropic plasticity models and some inherent complexity of the proposed material model.

\subsection{Orthotropic behaviour in uniaxial tension}

The ability of the proposed model to represent tensile orthotropic behaviour is now discussed. For this purpose, we consider a single element test, with dimensions $100 \times 100 \mathrm{~mm}^{2}$, under pure uniaxial tension. The material properties listed in Table I are assumed, where all the material parameters in the $y$ direction are reduced by a factor two (except the fracture energy) to simulate orthotropic behaviour. For the fracture energy $G_{\mathrm{f} y}$, three different values are considered: $0.3 G_{\mathrm{f} x}$ (general case), $0.5 G_{\mathrm{f} x}$ (isotropic softening) and $+\infty$ (elastic-perfectly-plastic behaviour).

The adopted values for the material parameters demonstrate that completely different behaviour along the two material axes can be reproduced, see Figure 5. Further insight into the behaviour of the model can be obtained from Figure 6, which shows the evolution of the tensile yield contour in the plane $\tau_{x y}=0$ upon increasing softening. In the first example, the material strength in the $y$ direction degrades at a faster rate than the material strength in the $x$ direction. The second example yields isotropic softening, which means that degradation of $f_{\mathrm{t} x}$ sub and $f_{\mathrm{t} y}$ occurs at the same rate, i.e the ratio of strength along the material axes $f_{\mathrm{t} x} / f_{\mathrm{t} y}$ is constant during any load history. In both examples, once the fracture energies are exhausted, a no-tension material is recovered, see Figures 6(a) and 6(b). Finally, the third example yields elastic-perfectlyplastic behaviour in the $y$ direction while softening is allowed to occur in the $x$ direction. This means that the ratio of strength along the material axes tends to zero and the yield surface is only allowed to shrink along the $x$-axis, see Figure 6(c).

\subsection{Orthotropic behaviour in uniaxial compression}

The ability of the proposed model to represent compressive orthotropic behaviour is now discussed. For this purpose, we again consider a single element test, with dimensions $100 \times 100 \mathrm{~mm}^{2}$, under pure uniaxial compression. The material properties listed in Table II are assumed, where all the material parameters in the $y$ direction are reduced by a factor two (except the compressive fracture energy) to simulate orthotropic behaviour. For the compressive fracture

Table I. Material properties for uniaxial tension test $(\alpha=1 \cdot 0)$

\begin{tabular}{cccc}
\hline \multicolumn{3}{c}{ Material properties } \\
\hline$E_{x}$ & $10000 \mathrm{~N} / \mathrm{mm}^{2}$ & $E_{y}$ & $5000 \mathrm{~N} / \mathrm{mm}^{2}$ \\
$v_{x y}$ & $0 \cdot 2$ & $G_{x y}$ & $3000 \mathrm{~N} / \mathrm{mm}^{2}$ \\
$f_{\mathrm{t} x}$ & $1.0 \mathrm{~N} / \mathrm{mm}^{2}$ & $f_{\mathrm{t} y}$ & $0.5 \mathrm{~N} / \mathrm{mm}^{2}$ \\
& & & Example $10.006 \mathrm{Nmm} / \mathrm{mm}^{2}$ \\
$G_{\mathrm{f} x}$ & $0.02 \mathrm{Nmm} / \mathrm{mm}^{2}$ & $G_{\mathrm{f} y}$ & Example $20.01 \mathrm{Nmm} / \mathrm{mm}^{2}$ \\
& & & Example $3+\infty \mathrm{Nmm} / \mathrm{mm}^{2}$ \\
\hline
\end{tabular}




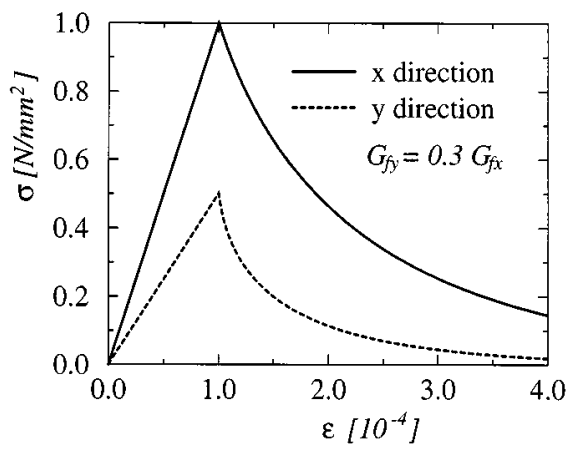

(a)

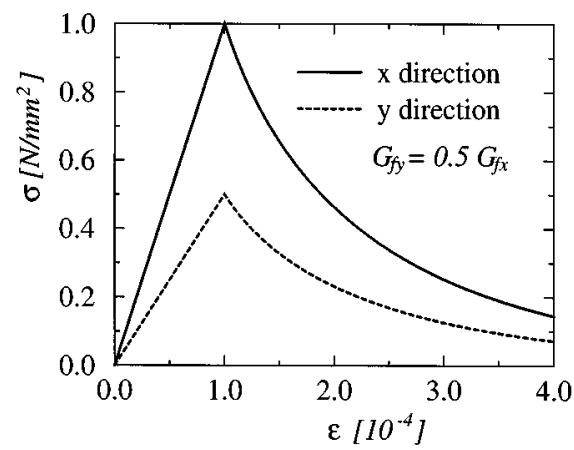

(b)

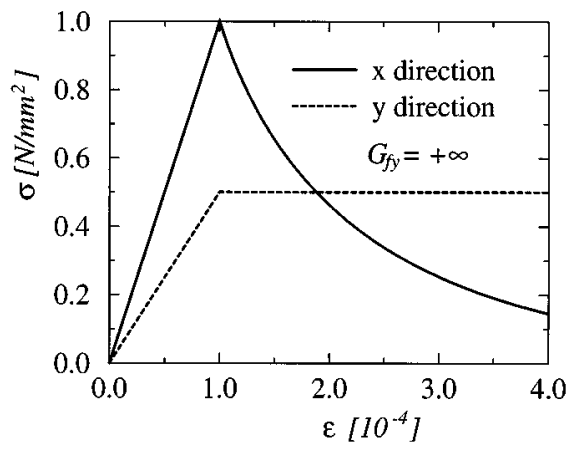

(c)

Figure 5. Stress-strain response in uniaxial tension along the two material axes: (a) example 1 (general case); (b) example 2 (isotropic softening); (c) example 3 (elastic-perfectly-plastic behaviour in $y$ direction)

energy $G_{\mathrm{fc} y}$, three different values are considered: $0.3 G_{\mathrm{fc} x}$ (general case), $0.5 G_{\mathrm{fc} x}$ (isotropic softening) and $+\infty$ (post-peak perfectly plastic behaviour).

Again, the values chosen for the material parameters illustrate the fact that completely different behaviour along the two material axes can be reproduced, see Figure 7. Further insight into the behaviour of the model can be obtained from Figure 8, which shows the evolution of the compressive yield contour in the plane $\tau_{x y}=0$ upon increasing softening. In the first example, the material strength in the $y$ direction degrades at a faster rate than the material strength in the $x$ direction. In the second example, degradation of the material strength in both directions occurs with the same rate and isotropic softening is obtained. In both examples, the residual yield surface, retrieved once the fracture energies are exhausted, is scaled from the peak yield surface while maintaining its original shape. Finally, in the third example, degradation only occurs for the material strength in the $x$ direction, while a post-peak perfectly plastic behaviour is obtained in the $y$ direction. This means that the ratio of strength along the material axes tends to a constant quasi-zero value and the residual yield surface has a different shape than the original one.

Note that, in the case of isotropic softening, the post-peak stress-strain diagrams under uniaxial loading conditions along the two material axes, see Figure 7(b), are not scaled by a factor 


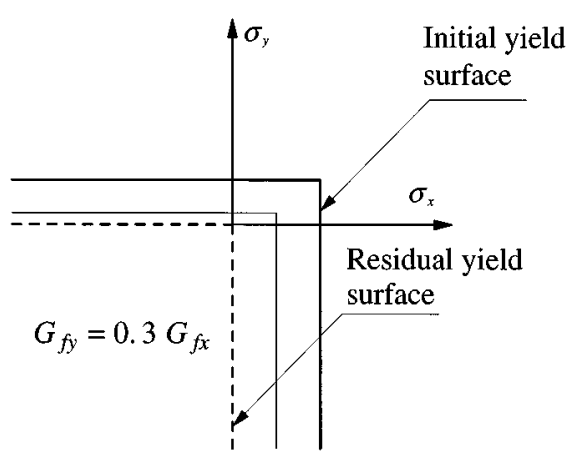

(a)

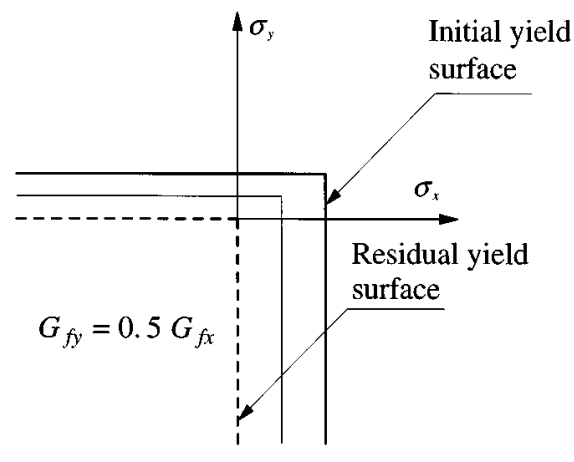

(b)

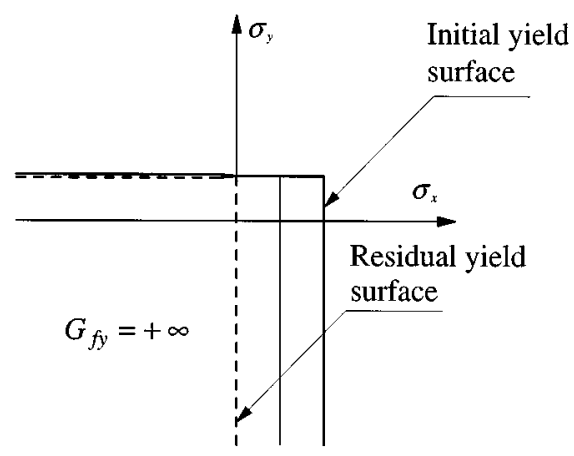

(c)

Figure 6. Evolution of the tensile criterion in the plane $\tau_{x y}=0$ upon increasing softening: (a) example 1 (general case); (b) example 2 (isotropic softening); (c) example 3 (elastic-perfectly-plastic behaviour in $y$ direction)

Table II. Material properties for uniaxial compression test $\left(\beta=-1 \cdot 0, \gamma=3.0\right.$ and $\left.\kappa_{\mathrm{p}}=0.0005\right)$

\begin{tabular}{lccc}
\hline \multicolumn{3}{c}{ Material properties } \\
\hline$E_{x}$ & $10000 \mathrm{~N} / \mathrm{mm}^{2}$ & $E_{y}$ & $5000 \mathrm{~N} / \mathrm{mm}^{2}$ \\
$v_{x y}$ & $0 \cdot 2$ & $G_{x y}$ & $3000 \mathrm{~N} / \mathrm{mm}^{2}$ \\
$f_{\mathrm{c} x}$ & $10.0 \mathrm{~N} / \mathrm{mm}^{2}$ & $f_{\mathrm{c} y}$ & $5 \cdot 0 \mathrm{~N} / \mathrm{mm}^{2}$ \\
& & & Example $11.5 \mathrm{Nmm} / \mathrm{mm}^{2}$ \\
$G_{\mathrm{fc} x}$ & $5 \cdot 0 \mathrm{Nmm} / \mathrm{mm}^{2}$ & $G_{\mathrm{fc} y}$ & Example $22.5 \mathrm{Nmm} / \mathrm{mm}^{2}$ \\
& & & Example $3+\infty \mathrm{Nmm} / \mathrm{mm}^{2}$ \\
\hline
\end{tabular}

two. This is solely due to the definition of the softening scalar and the fact that the yield value $\bar{\sigma}_{\mathrm{c}}$ is not equal to the uniaxial strength along each material axis. This also means that the definition of the 'compressive fracture energy' can be questioned because a perfect equivalence in the stressstrain diagram is not obtained. This limitation of the model can be easily solved, for example, if 


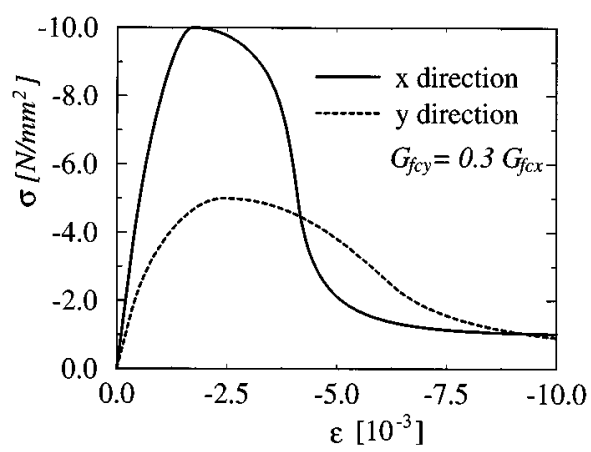

(a)

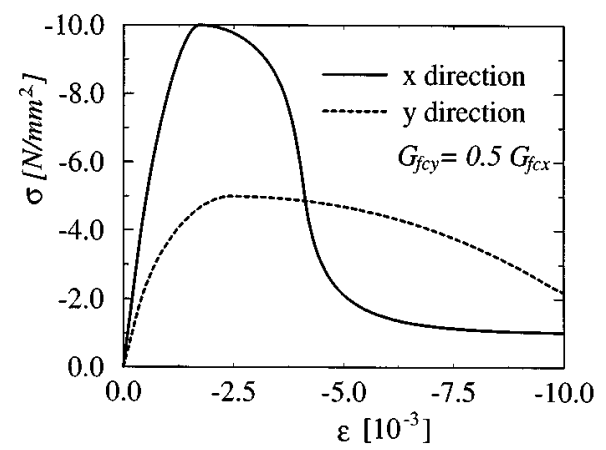

(b)

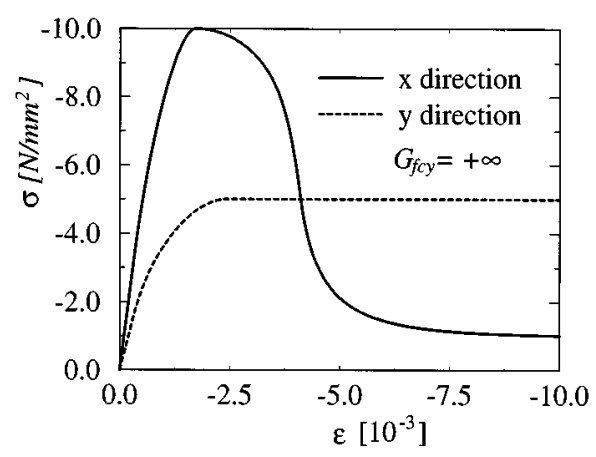

(c)

Figure 7. Stress-strain response in uniaxial compression along the two material axes: (a) example 1 (general case); (b) example 2 (isotropic softening); (c) example 3 (post-peak perfectly plastic behavior in $y$ direction)

a unit norm is used for the plastic flow vector and a strain softening hypothesis is used for the softening scalar. The additional difficulty introduced in the formulation $\left(\dot{\kappa}_{\mathrm{c}} \neq \dot{\lambda}_{\mathrm{c}}\right)$ is not particularly difficult to solve but, with the usual lack of experimental results on the material inelastic behaviour, the initial assumptions are advised in order to simplify the implementation.

\section{EXAMPLES}

As an example of the applicability of the model in the analysis of anisotropic structures, attention is given to tests carried out in masonry shear walls at ETH Zurich. ${ }^{26}$ These experiments in hollow clay brick masonry are well suited for the validation of the model because most of the material parameters are available from biaxial tests. Figure 9 shows the geometry of the walls, which consist of a masonry panel of $3600 \times 2000 \times 150 \mathrm{~mm}^{3}$ and two flanges of $150 \times 2000 \times 600 \mathrm{~mm}^{3}$. Additional boundary conditions are given by two concrete slabs placed in the top and bottom of the specimen. Initially, the wall is subjected to a vertical load $p$ uniformly distributed over the length of the wall. This is followed by the application of a horizontal force $F$ on the top slab causing a horizontal displacement $d$. 


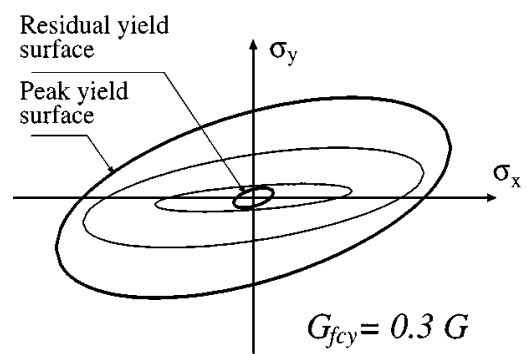

(a)

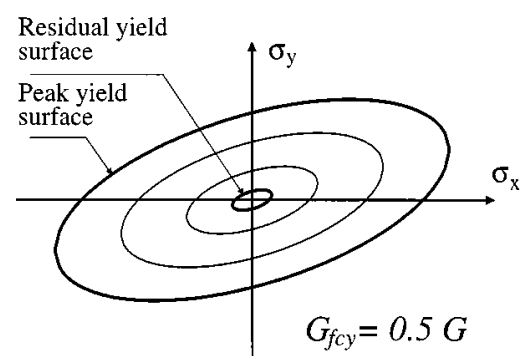

(b)

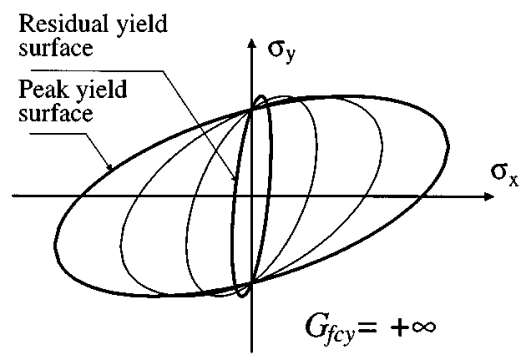

(c)

Figure 8. Evolution of the compressive criterion in the plane $\tau_{x y}=0$ upon increasing softening: (a) example 1 (general case); (b) example 2 (isotropic softening); (c) example 3 (post-peak perfectly plastic behaviour in $y$ direction)

A regular mesh of $24 \times 15$ four-noded quadrilaterals with full integration is used for the panel and $2 \times 15$ cross diagonal patches of three-noded triangles are used for each flange. It is noted that hybrid or mixed-type finite elements have not been employed on purpose because the danger of spurious modes in non-linear calculations ${ }^{27}$ is far more important than computing the shear stress wrongly in a linear elastic analysis with regular elements. The analyses are carried out with indirect displacement control with line searches, and the snap-backs are traced with COD control over the most active crack. It is noted that the self-weight of the wall and the top slab is also considered in the analyses. Two walls from the experiments, W1 and W2, are analysed with the composite plasticity model. The properties of the composite material are obtained from Ganz and Thürlimann, ${ }^{28}$ see Tables III and IV. Note the extreme anisotropy of the material, which is not due to the arrangement of the units and joints but it is due to the anisotropy of the hollow clay bricks. The axes of orthotropy $x-y$ coincide with the horizontal (bed) joints and vertical (head) joints, respectively. Nevertheless, it is noted that the flanges have the width of a single unit and the failure in the $x$ direction (equivalent to the out-of-plane direction of the panel) is determined by the tensile and compressive strength of the clay brick: $f_{\mathrm{t} x}=0.68 \mathrm{~N} / \mathrm{mm}^{2}$ and $f_{\mathrm{c} x}=9.5 \mathrm{~N} / \mathrm{mm}^{2}$.

The first wall, denoted by $\mathrm{W} 1$, is subjected to an initial vertical load $p$ of $0.61 \mathrm{~N} / \mathrm{mm}^{2}$. The comparison between numerical and experimental load-displacement diagrams, for wall $\mathrm{W} 1$, is given in Figure 10. Good agreement is found. The low initial vertical load combined with the confinement provided by the flanges and the top concrete slab yields a very ductile behaviour. The unloading found at $d \approx 2 \cdot 0 \mathrm{~mm}$ is due to the mode I crack opening of the left flange. 

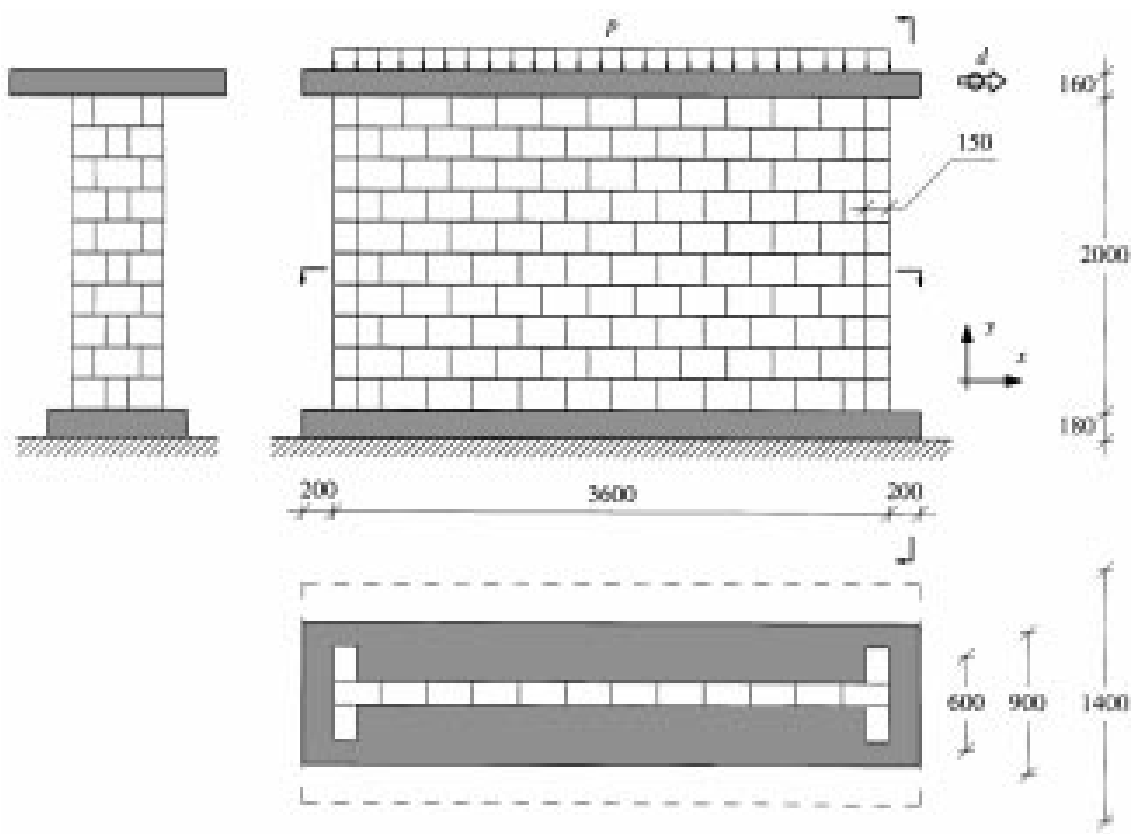

Figure 9. Geometry and loads for ETH Zurich shear walls

Table III. Walls W. elastic properties

\begin{tabular}{lccc}
\hline$E_{x}$ & $E_{y}$ & $v_{x y}$ & $G_{x y}$ \\
\hline 2460 & 5460 & $0 \cdot 18$ & 1130 \\
$\mathrm{~N} / \mathrm{mm}^{2}$ & $\mathrm{~N} / \mathrm{mm}^{2}$ & & $\mathrm{~N} / \mathrm{mm}^{2}$ \\
\hline
\end{tabular}

Table IV. Walls W. inelastic properties

\begin{tabular}{|c|c|c|c|c|c|c|}
\hline \multicolumn{7}{|c|}{ Tension regime } \\
\hline$f_{\mathrm{t} x}$ & $f_{\mathrm{t} y}$ & $\alpha$ & $G_{\mathrm{f} x}$ & $G_{\mathrm{f} y}$ & & \\
\hline $\begin{array}{l}0 \cdot 28 \\
\mathrm{~N} / \mathrm{mm}^{2}\end{array}$ & $\begin{array}{c}0.05 \\
\mathrm{~N} / \mathrm{mm}^{2}\end{array}$ & 1.73 & $\begin{array}{c}0.02 \\
\mathrm{Nmm} / \mathrm{mm}^{2}\end{array}$ & $\begin{array}{c}0.02 \\
\mathrm{Nmm} / \mathrm{mm}^{2}\end{array}$ & & \\
\hline \multicolumn{7}{|c|}{ Compression regime } \\
\hline$\overline{f_{\mathrm{c} x}}$ & $f_{\mathrm{c} y}$ & $\beta$ & $\gamma$ & $G_{\mathrm{fc} x}$ & $G_{\mathrm{fc} y}$ & $\kappa_{\mathrm{p}}$ \\
\hline $\begin{array}{l}1 \cdot 87 \\
\mathrm{~N} / \mathrm{mm}^{2}\end{array}$ & $\begin{array}{c}7.61 \\
\mathrm{~N} / \mathrm{mm}^{2}\end{array}$ & $-1 \cdot 05$ & $1 \cdot 20$ & $\begin{array}{c}5 \cdot 0 \\
\mathrm{Nmm} / \mathrm{mm}^{2}\end{array}$ & $\begin{array}{c}10 \cdot 0 \\
\mathrm{Nmm} / \mathrm{mm}^{2}\end{array}$ & $8 \times 10^{-4}$ \\
\hline
\end{tabular}

The behaviour of the wall at ultimate stage is depicted in Figure 11 in terms of deformed meshes and cracked Gauss points. The center node of the crossed diagonal patch of the flanges is not shown in order to obtain a more legible picture. Cracks are plotted normal to the tensile principal plastic strain directions with a thickness proportional to the tensile equivalent plastic 


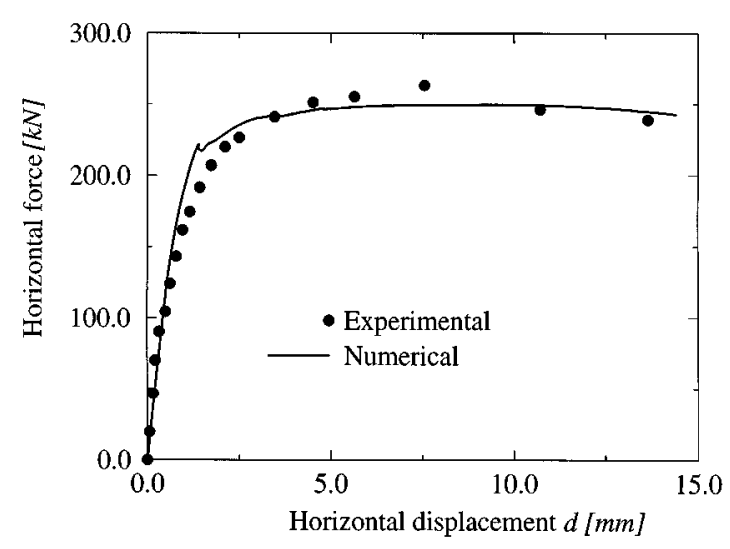

Figure 10. Wall W1. Load-displacement diagrams

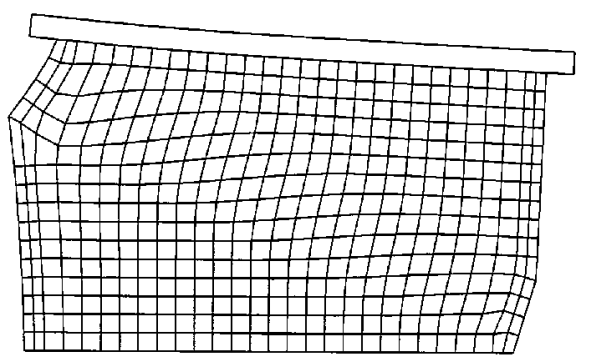

(a)

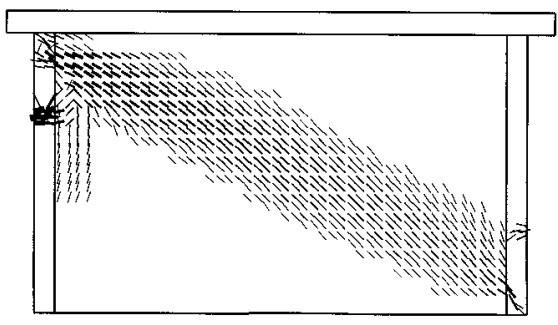

(b)

Figure 11. Wall W1. Results of the analysis at a displacement of 12.0 (mm) (ultimate): (a) deformed mesh; (b) cracks

strain. The cracks for which the tensile principal plastic strain fall in the lowest 5 per cent values have been discarded in order to obtain a more legible picture. At the ultimate stage, a well-defined failure mechanism is formed with a final band going from one corner of the specimen to the other and intersecting the flanges. This means that cracks rotate significantly after initiation into a sort of shear band, which agrees well with the experiments. ${ }^{26}$ Failure is exclusively governed by the tension regime.

The wall analysed next, denoted by $\mathrm{W} 2$, is subjected to an initial vertical load $p$ of $1.91 \mathrm{~N} / \mathrm{mm}^{2}$. The comparison between numerical and experimental load-displacement diagrams is given in Figure 12. From a qualitative perspective good agreement is found because the same trend is observed in both diagrams. Remarkably, the explosive type of failure observed in the experiments at a displacement of approximately $8.0 \mathrm{~mm}$ is also predicted by the analysis. Less good agreement is found with respect to the calculated collapse load value, which is 20 per cent higher than the experimental value. It is likely that the difference can be explained by the variation of the material properties in compression between the biaxial tests and the wall.

The behaviour of the wall W2 at the ultimate stage, see Figure 13 for deformed meshes and crack patterns, is quite different from the behaviour of wall W1. Two crack bands form which do not concentrate in a single band because crushing of the bottom-right corner of the structure 


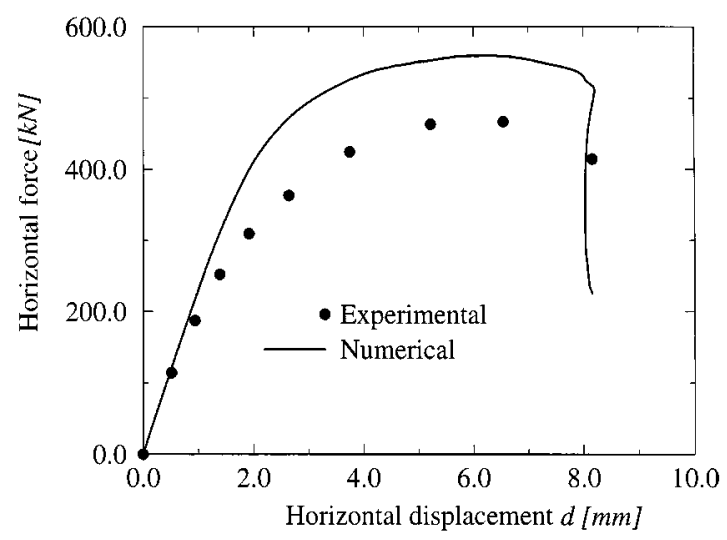

Figure 12. Wall W2. Load-displacement diagrams

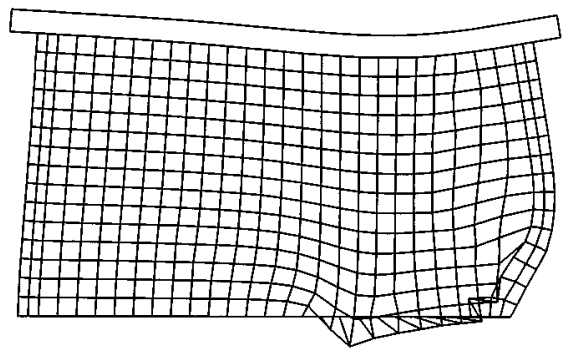

(a)

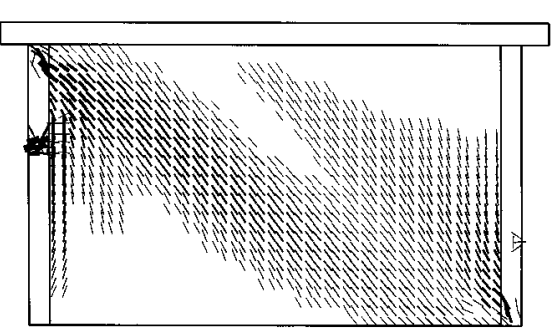

(b)

Figure 13. Wall W2. Results of the analysis at a displacement of 8.0 (mm) (ultimate): (a) (incremental) deformed mesh; (b) cracks

occurs first. This explosive type of failure, which is also observed in the experiments, ${ }^{26}$ has been traced with arc-length control over the nodes in the bottom row of elements of the panel. Failure is clearly governed by masonry crushing where, at the ultimate stage, the compressive strength of the bottom-right corner of the panel is completely exhausted.

\section{CONCLUSION}

An anisotropic composite continuum model for plane stress structures has been formulated in a modern computational plasticity format. It is assumed that two failure mechanisms can be distinguished, one associated with localised fracture processes and one associated with a more distributed fracture process which can be related to crushing of the material. The internal damage due to these failure mechanisms can be represented with two internal parameters $\kappa_{\mathrm{t}}$ and $\kappa_{\mathrm{c}}$ for damage in tension and compression, respectively. The model is formulated in such a way that each internal parameter is related to two independent fracture energies along each material axis. For this reason, it is possible to reproduce different inelastic behaviour along two orthogonal directions. 
The softening model features a Rankine-type criterion for tension and a Hill-type criterion for compression. A crucial point in the analysis of strain softening materials with a standard continuum is the sensitivity of the results with respect to the mesh size. The fracture energy-based regularization which has been adopted in this study is widely used in engineering practice to overcome this problem. It has been shown elsewhere ${ }^{18}$ that mesh independent results are, indeed, obtained with the proposed material model.

Good agreement has been found in a comparison between numerical results and experimental data for masonry shear walls where both modes of the composite model (tension and compression) become active. The model has also proved to be quite robust because it was possible to follow the complete load-deformation path until total degradation of the strength.

\section{ACKNOWLEDGMENTS}

This research has been supported financially by the Netherlands Technology Foundation (STW) under grant DCT 33.3052.

\section{REFERENCES}

1. R. Hill, 'A theory of the yielding and plastic flow of anisotropic metals', Proc. Roy. Soc., (London) A193, 281-288 (1948).

2. O. Hoffman, 'The brittle strength of orthotropic materials', J. Composite Mat., 1, 200-206 (1967).

3. S. W. Tsai and E. M. Wu, 'A general theory of strength of anisotropic materials', J. Composite Mat., 5, 58-80 (1971).

4. R. de Borst and P. H. Feenstra, 'Studies in anisotropic plasticity with reference to the Hill criterion', Int. J. Numer. Meth. Engng., 29, 315-336 (1990).

5. J. C. J. Schellekens and R. de Borst, 'The use of the Hoffman yield criterion in finite element analysis of anisotropic composites', Comput. Struct., 37, 1087-1096 (1990).

6. J. F. Besseling, 'A theory of elastic, plastic and creep deformations of an initially isotropic material showing anisotropic strain-hardening, creep recovery and secondary creep', J. Appl. Mech., 22, 529-536 (1958).

7. C. C. Swan and A. S. Cakmak, 'A hardening orthotropic plasticity model for non-frictional composites: rate formulation and integration algorithm', Int. J. Numer. Meth. Engng., 37, 839-860 (1994).

8. X. Li, P. G. Duxbury and P. Lyons, 'Considerations for the application and numerical implementation of strain hardening with the Hoffman yield criterion', Comput. Struct., 52, 633-644 (1994).

9. P. H. Feenstra and R. de Borst, 'A composite plasticity model for concrete', Int. J. Solids Struct., 33, 707-730 (1996).

10. S. Pietruszczak and Z. Mróz, 'Finite element analysis of deformation of strain-softening materials', Int. J. Numer. Meth. Engng., 17, 327-334 (1981)

11. Z. P. Bažant and B. H. Oh, 'Crack band theory for fracture of concrete', Mater. Struct., RILEM 93, 155-177 (1983).

12. K. J. Willam, 'Experimental and computational aspects of concrete fracture', in N. Bicanic et al., (eds.), Computer Aided Analysis and Design of Concrete Structures, Pineridge Press, 1984, pp. 33-70.

13. K. J. Willam, B. Hurlbut and S. Sture, 'Experimental and constitutive aspects of concrete failure', in C. Meyer and H. Okamura, (eds.), Finite Element Analysis of Reinforced Concrete Structures, ASCE, New York, 1986, pp. $226-245$.

14. R. A. Vonk, 'Softening of concrete loaded in compression', Dissertation, Eindhoven University of Technology, Eindhoven, The Netherlands, 1992

15. M. A. Crisfield, 'No-tension analysis of reinforced concrete beams with and without compressive softening', in D. R. J. Owen et al. (eds.), Computational Plasticity, Pineridge Press, 1989, pp. 1235-1250.

16. J. G. Rots, 'Computational modeling of concrete fracture', Dissertation, Delft University of Technology, Delft, The Netherlands, 1988.

17. J. C. Simo, J. G. Kennedy and S. Govindjee, 'Non-smooth multisurface plasticity and viscoplasticity. Loading/unloading conditions and numerical algorithms', Int. J. Numer. Meth. Engng., 26, 2161-2185 (1988).

18. P. B. Lourenço, 'Computational strategies for masonry structures', Dissertation, Delft University of Technology, Delft, The Netherlands, 1996.

19. W. T. Koiter, 'Stress-strain relations, uniqueness and variational theorems for elastic-plastic materials with a singular yield surface', Quart Appl. Math., 11, 350-354 (1953).

20. M. Ortiz and E. P. Popov, 'Accuracy and stability of integration algorithms for elastoplastic constitutive relations', Int. J. Numer. Meth. Engng., 21, 1561-1576 (1985). 
21. E. Pramono and K. Willam, 'Implicit integration of composite yield surfaces with corners', Engng. Comput., 6, 186-197 (1989).

22. J. C. Simo and R. L. Taylor, 'Consistent tangent operators for rate-independent elastoplasticity', Comp. Meth. Appl. Mech. Engng., 48, 101-118 (1985).

23. P. H. Feenstra and R. de Borst, 'A plasticity model and algorithm for mode-I cracking in concrete', Int. J. Numer. Meth. Engng., 38, 2509-2529 (1995).

24. P. B. Lourenço, J. G. Rots and P. H. Feenstra, 'A 'tensile' Rankine type orthotropic model for masonry', in G. N. Pande and J. Middleton (eds.), Computer Methods in Structural Masonry, Vol. 3, Books and Journals International, Swansea, UK, 1995, pp. 167-176.

25. P. B. Lourenço, 'An orthotropic continuum model for the analysis of masonry structures', Report 03-21-1-31-27, Delft University of Technology, Delft, The Netherlands, 1995.

26. H. R. Ganz and B. Thürlimann, 'Tests on masonry walls under normal and shear loading (in German)', Report No. 7502-4, Institute of Structural Engineering, ETH Zurich, Zurich, Switzerland, 1984.

27. R. de Borst and A. E. Groen, 'Some observations on element performance in isochoric and dilatant plastic flow', Int. J. Numer. Meth. Engng., 38, 2887-2906 (1995).

28. H. R. Ganz and B. Thürlimann, 'Tests on the biaxial strength of masonry (in German)', Report No. 7502-3, Institute of Structural Engineering, ETH Zurich, Zurich, Switzerland, 1982. 